\title{
Rat Notochordal Cells Undergo Premature Stress-Induced Senescence by High Glucose
}

\author{
Jong-Beom Park, Chu-Hwan Byun, Eun-Young Park \\ Department of Orthopedic Surgery, Uijeongbu St. Mary's Hospital, College of Medicine, The Catholic University of Korea, Uijeongbu, Korea
}

\begin{abstract}
Study Design: In vitro cell culture.
Purpose: The purpose of the study was to investigate the effect of high glucose on premature stress-induced senescence of rat notochordal cells.

Overview of Literature: Glucose-mediated increase of oxidative stress is a major causative factor for the development of diseases associated with diabetes mellitus such as senescence. However, no information is available for the effect of high glucose on premature stress-induced senescence of rat notochordal cells.

Methods: Notochordal cells were isolated from 4-week-old rats, cultured and placed in either $10 \%$ fetal bovine serum (FBS, normal control) or $10 \%$ FBS plus two high glucose concentrations (0.1 M and $0.2 \mathrm{M}$, experimental conditions) for 1 and 3 days. We identified and quantified the mitochondrial damage (mitochondrial transmembrane potential), reactive oxygen species (ROS) and antioxidants, such as manganese superoxide dismutase (MnSOD) and catalase, for each condition. We also identified and quantified senescence and telomerase activity. Finally, we determined the expression of proteins related to replicative senescence (p53-p21-pRB) and stressinduced senescence (p16-pRB) pathways.

Results: Two high glucose concentrations enhanced the disruption of mitochondrial transmembrane potential and excessive generation of ROS in notochordal cells for 1 and 3 days, respectively. The expressions of MnSOD and catalase were increased in notochordal cells treated with both high glucose concentrations at 1 and 3 days. The telomerase activity declined at 1 and 3 days. Two high glucose concentrations increased the occurrence of stress-induced senescence of notochordal cells by p16-pRB pathways at 1 and 3 days.

Conclusions: Despite compensatory expression of antioxidants, high glucose-induced oxidative stress accelerates stress-induced senescence in rat notochordal cells. This may result in dysfunction of notochordal cells, leading to accelerated premature disc degeneration. The prevention of excessive generation of oxidative stress by strict blood glucose control is important to prevent or to delay premature disc degeneration in young patients with diabetes mellitus.
\end{abstract}

Keywords: High glucose; Stress-induced senescence; Notochordal cells; Intervertebral disc degeneration

\section{Introduction}

Cellular senescence is an irreversible growth arrest that can occur after repeated cell division (replicative senes- cence). It can also be prematurely induced by stress conditions such as oxidative stress, DNA damage and mitogenic stress (stress-induced senescence) [1-3]. Senescent cells cannot divide even if stimulated by mitogens, but

Received Dec 10, 2014; Revised Jan 9, 2015; Accepted Jan 12, 2015

Corresponding author: Jong-Beom Park

Department of Orthopedic Surgery, Uijeongbu St. Mary's Hospital, College of Medicine,

The Catholic University of Korea, 271 Cheonbo-ro, Uijeongbu 480-717, Korea

Tel: +82-31-820-3578, Fax:+82-31-847-3671, E-mail: spinepjb@catholic.ac.kr 
they are not physiologically inert displaying altered phenotypic changes and gene expression. Furthermore, they secrete degradative enzymes, inflammatory cytokines, and growth factors, leading to a shift from extracellular matrix synthesis to degradation. Therefore, senescence is considered as a major factor contributing to age- or diseaseassociated tissue dysfunction and pathology. Replicative senescence is mediated by the p53-p21-pRB pathway; whereas stress-induced senescence is mediated by the p16-pRB pathway [4-6]. Senescent cells are characterized by telomere shortening and decreased telomerase activity. In addition, senescence-associated-beta-galactosidase (SA- $\beta$-Gal) activity is used to identify the occurrence of senescence [7-9].

Diabetes mellitus (DM) is a major public health problem worldwide. The number of DM patients is projected to reach 300 million in 2025 (International Diabetes Federation, 2001) [10]. Untreated DM can cause many complications, such as cardiovascular disease, chronic renal failure, retinopathy and neuropathy [11]. DM is also considered an important etiological factor in disc degeneration [12-14]. Previous studies have reported a higher incidence of degenerative disc diseases in patients with DM than in the patients without DM [15]. A considerable portion of patients who undergo spine surgery suffer from DM. Their surgical outcome was reported to be poor, as compared to non-DM patients $[16,17]$. Recently, there have been reports on the association of senescence and disc degeneration [18-23]. Premature disc degeneration is accompanied by the loss and/or dysfunction of notochordal cells. However, senescence of notochordal cells as a biological mechanism and the role of senescence in disc degeneration are not well understood to date.

The glucose-mediated increase in oxidative stress, such as the reactive oxygen species (ROS), is a main biochemical pathway in diseases associated with DM [24-26]. The mitochondria is the main source of endogenous ROS in most mammalian cell types [27]. ROS play a role in various cellular processes, such as senescence, which appear to cause cellular damage and physiological dysfunction. Therefore, the accumulation of ROS is reportedly associated with a variety of diseases including neurodegenerative diseases, DM, cancer, premature aging, and inflammatory disorders $[28,29]$. To date, little information is available about the effect of DM on the senescence of notochordal cells and premature disc degeneration. It is important to clarify the effect of DM on the senescence of notochordal cells to prevent or to delay premature disc degeneration in young patients with DM. We therefore investigated the effect of DM on mitochondrial damage, oxidative stress, senescence of nucleus pulposus (NP) cells, and premature disc degeneration.

\section{Materials and Methods}

\section{Rat notochordal cells culture and high glucose treat- ments}

All lumbar intervertebral discs (L1-6) were harvested from 4-week-old male Sprague Dawley rats (Orient Bio., Seoul, Korea) immediately after sacrifice. The discs were carefully dissected under a microscope to obtain only the gelatinous NP tissue, and the harvested NP tissue was pooled in $\alpha$-minimum essential medium ( $\alpha$-MEM, Gibco BRL, Grand Island, NY, USA). The cells were released from the NP tissue in Hank's Balanced Salt Solution (Hyclone, Ottawa, ON, Canada) with $0.02 \%$ pronase (SigmaAldrich, St. Louis, MO, USA) by vigorous pipetting. After the cells grew to confluence, they were split once (passage 1) and allowed to reach confluence again. When the cells reached $80 \%-90 \%$ confluence they were placed in either $10 \%$ fetal bovine serum (FBS; normal control) or $10 \%$ FBS plus two different high glucose concentrations $(0.1 \mathrm{M}$ and $0.2 \mathrm{M}$, experimental conditions) for 1 and 3 days. The study was approved by the Institution's Animal Care and Use Committee.

\section{Detection of mitochondrial damage}

Mitochondrial damage of notochordal cells was detected with the mitochondrial transmembrane potential apoptosis detection kit (Abcam Plc, Cambridge, UK). The kit utilizes MitoCapture a cationic dye that differentially fluoresces in healthy and damaged apoptotic cells. In damaged apoptotic cells, MitoCapture cannot aggregate in the mitochondria due to the altered mitochondrial transmembrane potential, and thus it remains in the cytoplasm in its monomeric form, fluorescent green. The cells were seeded on a glass cover slip in 12-well culture plates and then incubated with the MitoCapture reagent in a prewarmed incubation buffer in a humidified atmosphere for 30 minutes at $37^{\circ} \mathrm{C}$. Fluorescent signals were detected with a fluorescence microscope (Image-Pro Plus, Media Cybernetics, Rockville, MD, USA; Olympus CO., Tokyo, Japan). 


\section{Intracellular ROS measurement}

Intracellular ROS accumulation was measured by ROS detection with $\mathrm{H}_{2}$ DCF-DA (Sigma-Aldrich) according to the manufacturer's instructions. Notochordal cells were stained with $25 \mu \mathrm{M}$ of $\mathrm{H}_{2}$ DCF-DA in phosphate buffered saline (PBS) in a humidified atmosphere (95\% air, 5\% $\mathrm{CO}_{2}$ ) for 30 minutes at $37^{\circ} \mathrm{C}$. Fluorescence intensity was measured with BD FACS Canto flow cytometer (BD Biosciences, Franklin Lakes, NJ, USA).

\section{Expression of manganese superoxide dismutase (MnSOD)}

Notochordal cells were incubated with rabbit anti-MnSOD (Millipore Co., Millipore Billerica, MA, USA) antibody in a blocking solution for 1 hour at room temperature. After washing, fluorescence-conjugated Cy3-Donkey anti-rabbit antibody (Millipore Co.) and Hoechst Nuclear HCS solution (Millipore Co.) in blocking solution were added and the cells were incubated for 1 hour in the dark. After further washing steps, characteristic markers of organelle phenotype were determined by fluorescence microscopy (Image-Pro Plus, Olympus).

\section{Expression of catalase}

The intracellular production of catalase by notochordal cells was measured with the Catalase Assay Kit (Cayman Chemical Co., Ann Arbor, MI, USA) according to the manufacturer's instructions. The cells were sonicated on ice in $50 \mathrm{mM}$ potassium phosphate, $\mathrm{pH} 7.0$ containing $1 \mathrm{mM}$ ethylenediaminetetraacetic acid. Absorbance was measured at $540 \mathrm{~nm}$ using a plate reader (Molecular Devices, Sunnyvale, CA, USA).

\section{Determination of senescence}

The SA- $\beta$-Gal activity in the notochordal cells was determined using a SA- $\beta$-Gal staining kit (Cell Signaling Technology). The cells were plated on to poly (L-lysine)-coated 12 -mm glass cover slips and fixed with $2 \%$ formaldehyde and $0.2 \%$ glutaraldehyde in PBS for 15 minutes at room temperature. The slides were rinsed with PBS and then incubated for 8 hours at $37^{\circ} \mathrm{C}$ with fresh SA- $\beta$-Gal staining solution containing $40 \mathrm{mM}$ citric acid/sodium phosphate (pH 6.0), $150 \mathrm{mM} \mathrm{NaCl}, 2 \mathrm{mM} \mathrm{MgCl}, 5 \mathrm{mM}$ potassium ferrocyanide, $5 \mathrm{mM}$ potassium ferricyanide, and $1 \mathrm{mg} / \mathrm{mL}$ of 5-bromo-4-chloro-3-indolyl- $\beta$-D-galactopyranoside (X-gal). After rinsing with $70 \%$ glycerol, blue-stained cells were identified as senescent cells under a microscope. All notochordal cells and SA- $\beta$-Gal-positive notochordal cells on the entire section were counted $(\times 200)$ and the percentage of SA- $\beta$-Gal-positive notochordal cells was calculated.

\section{Determination of telomerase activity}

The telomerase activity was analyzed using a TeloTAGGG Telomerase PCR ELISA Plus Kit (Roche, Mannheim, Germany), according to the manufacturer's instructions. Using the ELISA method, the amplified products were immobilized on streptavidin-coated microtiter plates via biotin-streptavidin interaction. Thereafter the amplifications were detected by anti-digoxigenin antibodies conjugated to peroxidase. After addition of the peroxidase substrate (3, 3, 5, 5'-tetramethylbenzidine), the amount of TRAP products was determined by measurement of absorbance at $450 \mathrm{~nm}$ using a microplate reader.

\section{Expressions of $\mathrm{p} 53, \mathrm{p} 21, \mathrm{pRB}$, and $\mathrm{p} 16$}

Notochordal cells were plated on to the poly (L-lysin)coated $12 \mathrm{~mm}$ glass cover slips. The cells were fixed with 4\% paraformaldehyde in PBS for 15 minutes at room temperature, and washed with PBS ( $\mathrm{pH}$ 7.4). The cells were permeabilized with $0.2 \%$ TritonX-100 in PBS for 10 minutes. After incubation for 30 minutes at room temperature with PBS containing 3\% BSA (blocking solution), cells were incubated at $4^{\circ} \mathrm{C}$ overnight with the primary antibody (1:100 dilution) for p53 (Novus Biologicals, Littleton, CO, USA), p21 (Abcam Plc, Cambridge, UK), pRB (Biorbyt Ltd., Cambridge, UK), and p16 (Santa Cruz Biotechnology, Santa Cruz, CA, USA). After washing, the anti-rabbit Alexa Fluor 488 (Invitrogen, Carlsbad, CA, USA) and anti-mouse Alexa Fluor 555 (Invitrogen) fluorescence-conjugated IgG antibodies (1:5,000 dilution) in the blocking solution were added and incubated for 1 hour at room temperature in the dark. The cover slips were mounted on to the objective glasses with mounting medium containing 4, 6-diamidino-2-phenylindole (Vector, Burlingame, CA, USA). Fluorescent signals were detected by fluorescence microscopy (Olympus CO.). 


\section{Statistical analysis}

All experiments were independently conducted thrice, and the results were expressed as mean and standard deviation of the values derived with the three tests. Statistical analysis was done with the paired-samples $t$-test. A $p<0.05$ was considered as the level of significance.

\section{Results}

\section{Excessive generation of ROS through mitochondrial damage}

Immunofluorescence of rat notochordal cells treated with the two high glucose concentrations for 1 and 3 days demonstrated an enhanced disruption of the mitochondrial transmembrane potential (green color), which indicated mitochondrial damage, as compared to the control (Fig. 1). The expression of the mitochondrial transmembrane potential (green color) was detected in the nuclei of notochordal cells, whereas the expression of the mitochondrial transmembrane potential was observed in not only the nuclei of notochordal cells but also in the cytoplasm around the nuclei. Flow cytometry showed that $0.1 \mathrm{M}$ and $0.2 \mathrm{M}$ glucose enhanced ROS generation (\% total) in rat notochordal cells at 1 day ( $18.7 \%$ vs. $23.5 \%, p<0.05 ; 18.7 \%$ vs. $37.4 \%, p<0.01$ ), as compared to the normal control. Flow cytometry showed that $0.1 \mathrm{M}$ and $0.2 \mathrm{M}$ glucose en-

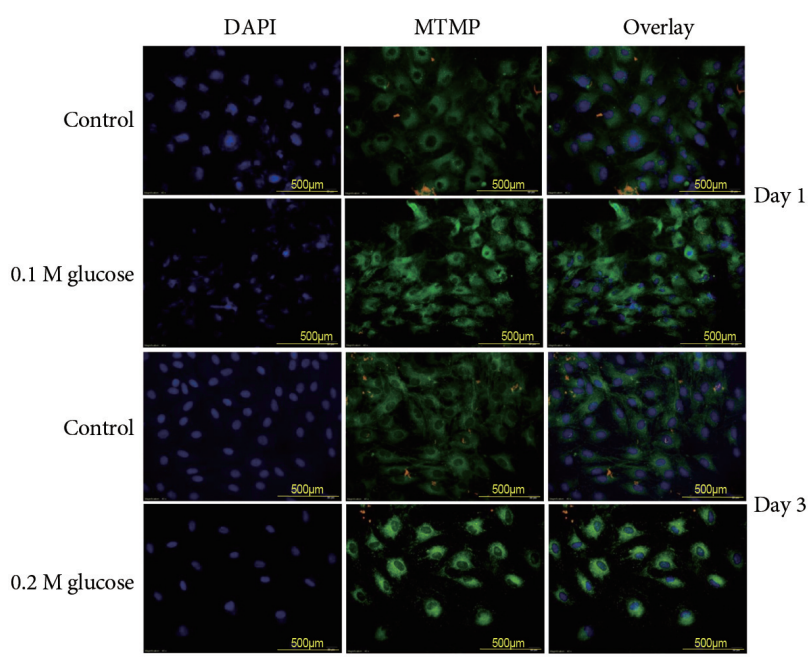

Fig. 1. Immunofluorescence demonstrated an enhanced disruption of the mitochondrial transmembrane potential (green color), which indicated mitochondrial damage in rat notochordal cells treated with the two high glucose concentrations at 1 and 3 days when compared to the control. hanced ROS generation ( $\%$ total) in rat NP cells at 3 days ( $30.8 \%$ vs. $39.1 \%, p<0.05 ; 30.8 \%$ vs. $42.8 \%, p<0.05$ ), as compared to the normal control.

\section{Enhanced MnSOD and catalase expressions}

The immunofluorescence demonstrated that the two high glucose concentrations enhanced compensatory expression (red color) of MnSOD in notochordal cells at 1 and 3 days, as compared with the control (Fig. 2). Expression of MnSOD (red color) was detected in the nuclei of notochordal cells, whereas expression of MnSOD was observed in not only the nuclei of notochordal cells but also in the cytoplasm around the nuclei. According to results of the Catalase Assay Kit (Cayman Chemical Co.), 0.1M and $0.2 \mathrm{M}$ glucose increased catalase expression (mmoL/ $\mathrm{min} / \mathrm{mL})$ in rat notochordal cells at 1 day (3.2 vs. 4.5 , $p<0.05 ; 3.2$ vs. $5.2, p<0.05)$, as compared to the normal control. In addition, $0.1 \mathrm{M}$ and $0.2 \mathrm{M}$ glucose increased expression of catalase $(\mathrm{mmoL} / \mathrm{min} / \mathrm{mL})$ in rat notochordal cells at 3 days ( 5.2 vs. $8.9, p<0.05 ; 5.2$ vs. $11.9, p<0.01$ ), as compared to normal control.

\section{Decreased telomerase activity}

$0.1 \mathrm{M}$ and $0.2 \mathrm{M}$ glucose decreased the mean telomerase activity (arbitrary units) in rat notochordal cells at 1 day ( 2.5 vs. $2.1, p<0.05 ; 2.5$ vs. $1.4, p<0.01$ ), as compared to

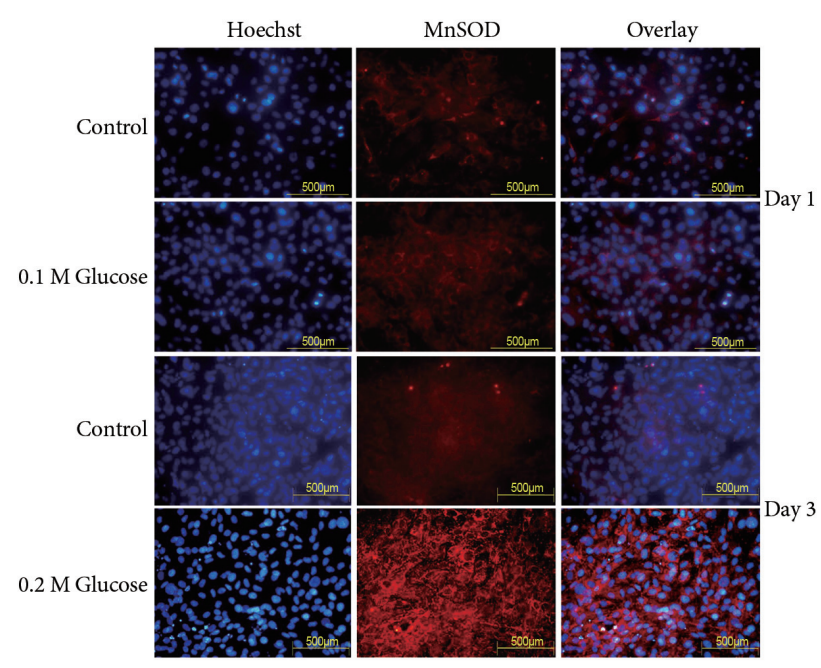

Fig. 2. Immunofluorescence demonstrated that the two high glucose concentrations enhanced compensatory expression (red color) of manganese superoxide dismutase (MnSOD) in rat notochordal cells at 1 and 3 days when compared with the control. 
the normal control. In addition, $0.1 \mathrm{M}$ and $0.2 \mathrm{M}$ glucose decreased the mean telomerase activity (arbitrary units) in rat notochordal cells at 1 day ( 1.6 vs. $0.7, p<0.01 ; 1.6$ vs. $0.5, p<0.01)$, as compared to the normal control.

\section{Increased stress-induced senescence of NP cells}

The mean percentage of SA- $\beta$-Gal-positive notochordal cells was significantly increased in notochordal cells treated with both high glucose concentrations for 1 and 3 days in a dose- and time-dependent manner, as compared with the control (Fig. 3). Immunofluorescence demonstrated that the two high glucose concentrations enhanced expression of p16 (Fig. 4) and pRB (Fig. 5) proteins in

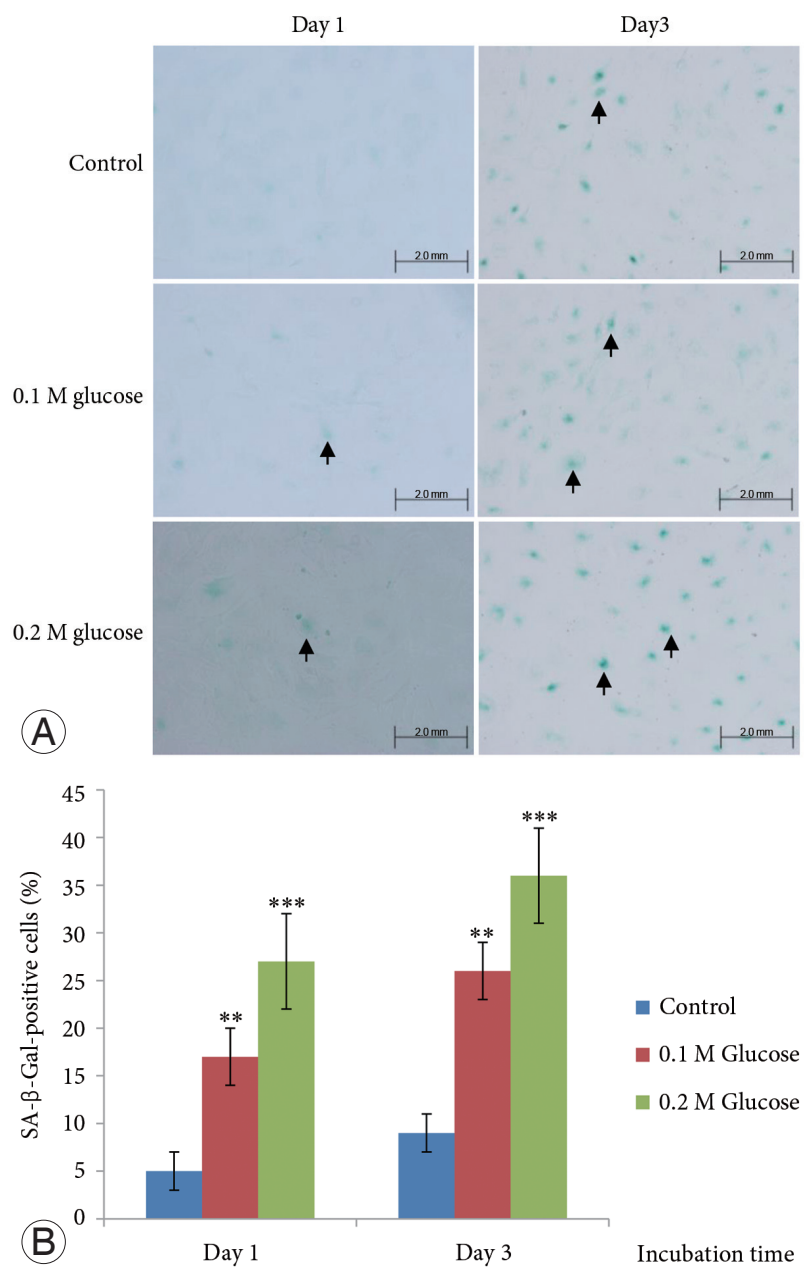

Fig. 3. (A) Both high glucose concentrations increased the occurrence of senescence in rat notochordal cells for 1 and 3 days, as compared to the control. (B) The mean percentage of senescence-associated-betagalactosidase (SA- $\beta$-Gal)-positive notochordal cells was significantly higher in rat notochordal cells treated with both high glucose concentrations when compared with the control. ${ }^{* *} p<0.001,{ }^{* *} p<0.01$. notochordal cells at 1 and 3 days when compared with the controls, respectively. However, immunofluorescence demonstrated that the two high glucose concentrations decreased the expression of p53 (Fig. 6) and p21 (Fig. 7) proteins in notochordal cells at 1 and 3 days when compared with the controls, respectively.

\section{Discussion}

The current findings demonstrated that both high glucose concentrations caused mitochondrial damage and consequently increased ROS generation in rat notochordal cells.

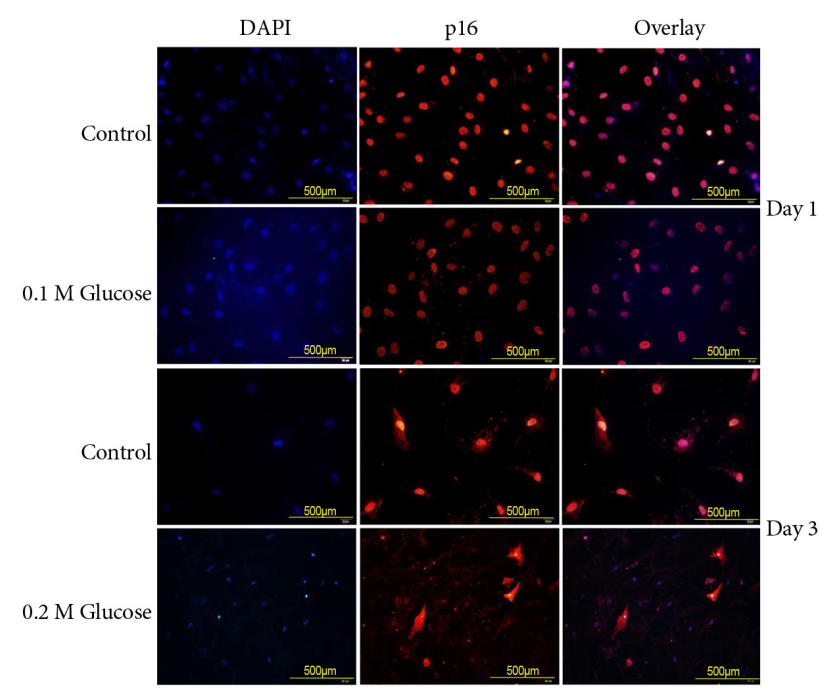

Fig. 4. Immunofluorescence demonstrated that the two high glucose concentrations enhanced expression of p16 (red color) protein in rat notochordal cells at 1 and 3 days when compared with the control.

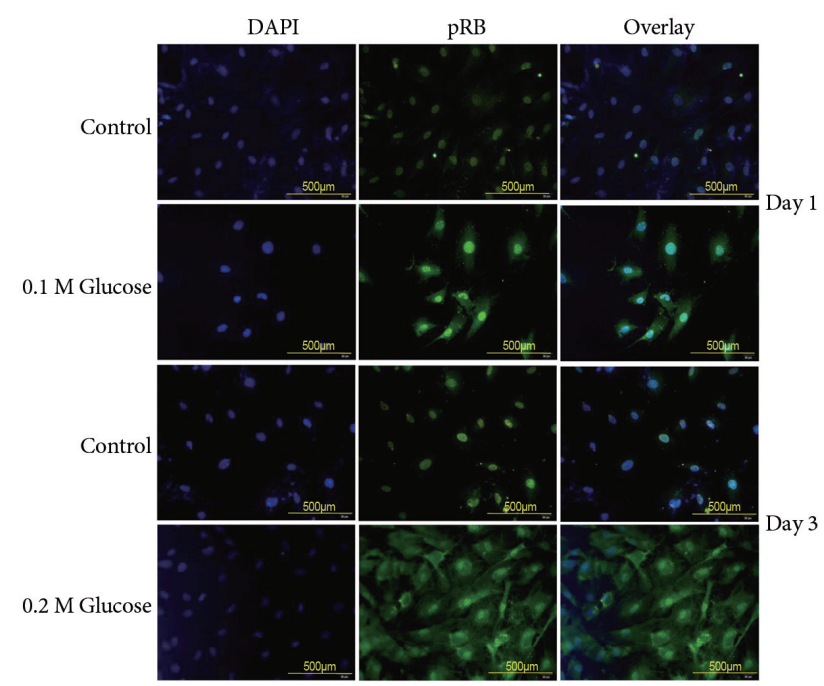

Fig. 5. Immunofluorescence demonstrated that the two high glucose concentrations enhanced expression of pRB (green color) protein in rat notochordal cells at 1 and 3 days when compared with the control. 


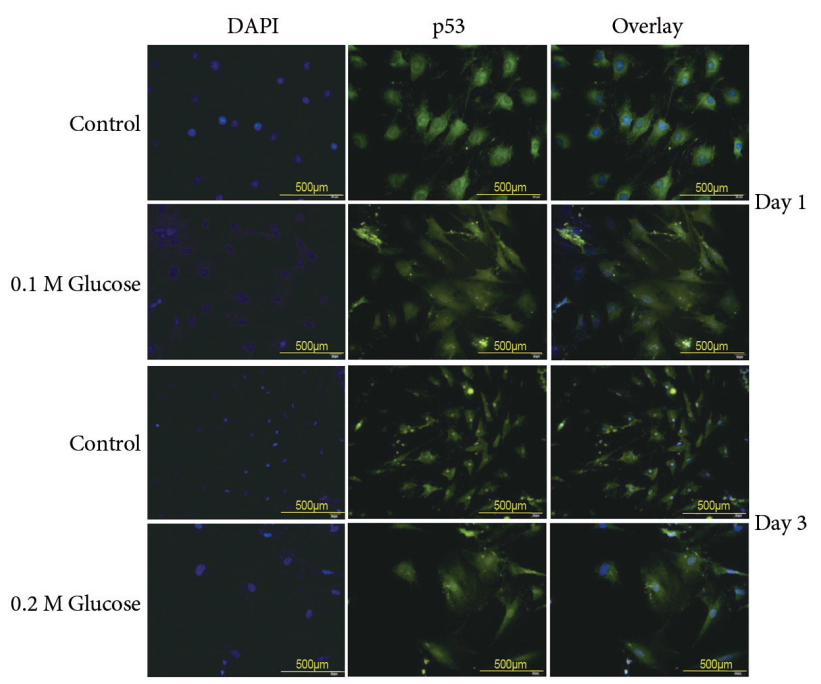

Fig. 6. Immunofluorescence demonstrated that the two high glucose concentrations decreased the expression of p53 protein in rat notochordal cells at 1 and 3 days when compared with the control.

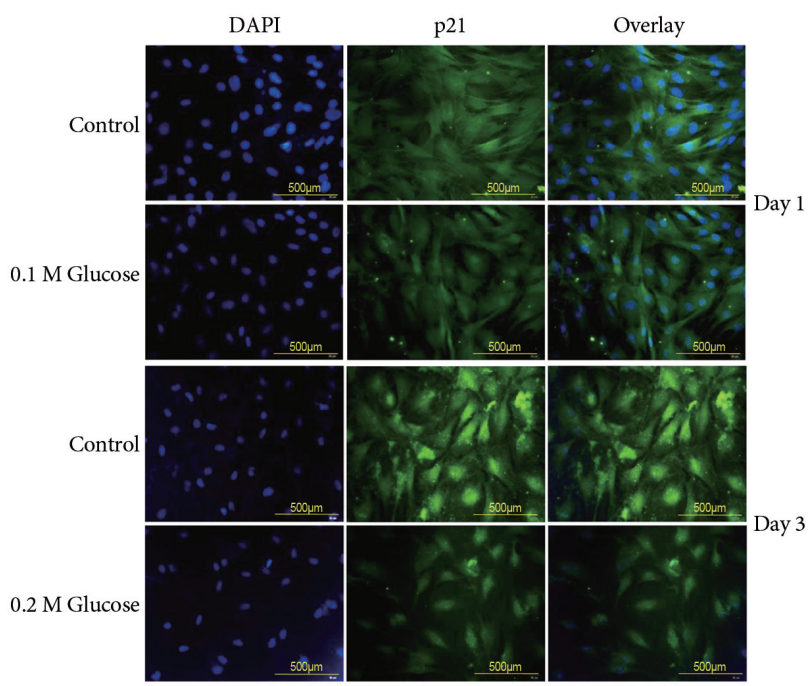

Fig. 7. Immunofluorescence demonstrated that the two high glucose concentrations decreased the expression of p21 protein in rat notochordal cells at 1 and 3 days when compared with the control.

The occurrence of senescence was significantly increased in notochordal cells treated with high glucose concentrations despite the compensatory expression of antioxidants, such as MnSOD and catalase. Telomerase activity declined in notochordal cells treated with both high glucose concentrations. The expressions of stress-induced senescence (p16-pRB) pathway were enhanced in notochordal cells treated with high glucose concentrations. However, the expressions of proteins related to replicative senescence (p53-p21) were decreased in notochordal cells treated with both concentrations of glucose. These results suggested that high glucose-induced oxidative stress accelerates the stress-induced senescence of notochordal cells through mitochondrial damage. This may result in dysfunction of notochordal cells, leading to accelerated premature disc degeneration. Thus, accelerated stress-induced senescence of notochordal cells could be an emerging risk factor for disc degeneration in young patients with DM. These results indicate the importance of strict blood glucose control in young patients with DM to prevent an excessive generation of oxidative stress and subsequently to prevent or delay premature disc degeneration.

Senescence may have evolved as a mechanism to prevent cells with damaged DNA from being replicated and thus to prevent tumor formation [30-32]. Replicative senescence is associated with changes in DNA structure and function including a telomere shortening and decreased telomerase activity [1-3]. However, senescence appears to be much more complex than a simple cell-cycle arrest occurring after a finite number of cell divisions. Stress-induced senescence can occur due to diverse stimuli including ultraviolet radiation, oxidative damage, activated oncogenes, and chronic inflammation [4-6]. Oxidative damage to DNA can directly contribute to stress-induced senescence and result in telomere shortening as seen in replicative senescence because the ends of chromosomes are particularly sensitive to oxidative damage [4-6]. It is well known that damaged mitochondria release harmful ROS into the cytosol [24-29]. Previous studies reported that antioxidants can protect harmful effects of oxidative stress and extend life-span and reduce age-related changes in tissues such as that of the heart [1-3]. In the current study, we demonstrated excessive oxidative stress, such as ROS release, through mitochondrial damage in rat notochordal cells treated with high glucose concentrations, as compared to the normal control. However, expression of antioxidants, such as MnSOD and catalase, were also increased in response to both high glucose concentrations, as compared to normal controls. These findings suggested the possibility of a cycle wherein an increased generation of ROS stress leads to compensatory production of antioxidants to neutralize oxidative stress. However, compensatory increased expression of antioxidants could not prevent or reduce the occurrence of premature stress-induced senescence of notochordal cells. We did not comparatively analyze the degree of oxidative stress (ROS) and antioxidants (MnSOD and catalase) in rat notochordal cells treated 
with high glucose. However, considering the accelerated stress-induced senescence in rat notochordal cells, it is likely that the excessive generation of oxidative stress in rat notochordal cells undergoing high glucose treatment supersedes the protective effect of antioxidants. Further studies are needed to confirm this speculation. Therefore, this result suggested that antioxidants therapy might be ineffective to prevent or delay premature disc degeneration in young patients with DM.

A hallmark of senescence is the expression of SA- $\beta-$ Gal. However, it is not strictly specific for senescent cells, since non-senescent cells with a high lysosomal content also stain positive in this assay [7-9]. The current study showed a higher percentage of SA- $\beta$-Gal-positive staining indicative of a higher rate of senescence in rat notochordal cells treated with both high glucose concentrations, as compared to normal control. To supplement shortcomings of SA- $\beta-\mathrm{Gal}$ staining, we additionally performed immunofluorescence staining to determine the expressions of proteins, such as p53, p21, pRB, and p16, related to replicative and stress-induced senescence pathways. Immunofluorescence showed that the senescent rat notochordal cells undergoing high glucose treatments significantly expressed p16 and pRB proteins, whereas the expressions of $\mathrm{p} 53$ and $\mathrm{p} 21$ proteins were significantly decreased, as compared to the normal control. We further showed that high glucose concentrations reduce the telomerase activity of rat notochordal cells. These results indicated that high glucose-induced oxidative stress predominantly accelerates premature stressinduced senescence (p16-pRB) in rat notochordal cells.

Prior to this study, it whether high glucose-induced oxidative stress caused diabetes-related notochordal cells' senescence was unknown. We evaluated the effects of high glucose concentrations on the induction of oxidative stress through mitochondrial damage and senescence in rat notochordal cells. Our study demonstrated that the percentage of senescent notochordal cells increased in proportion to the dose and time of high glucose treatment. This may explain the increased risk of disc degeneration with severity and duration of DM, which results in accelerated premature disc degeneration in young patients with DM. There were some limitations to the current study. Firstly, we did not investigate the telomere length. Telomere shortening is a characteristic finding for cellular senescence. Secondly, in vitro high glucose-induced diabetic model cannot perfectly reflect in vivo aspects of disc degeneration with DM, especially in terms of high glucose concentrations and culture period. Thus, further in vivo studies are needed to clarify these limitations.

\section{Conclusions}

The current study demonstrated that high glucoseinduced oxidative stress accelerates premature stressinduced senescence of rat notochordal cells despite the compensatory expression of antioxidants. This may result in dysfunction of notochordal cells, leading to accelerated premature disc degeneration. Thus, accelerated stress-induced senescence of notochordal cells could be an emerging risk factor for premature disc degeneration in young patients with DM. Therefore, strict blood glucose control could be important to prevent or to delay premature disc degeneration in young patients with DM.

\section{Conflict of Interest}

No potential conflict of interest relevant to this article was reported.

\section{References}

1. Tsirpanlis G. Cellular senescence, cardiovascular risk, and CKD: a review of established and hypothetical interconnections. Am J Kidney Dis 2008;51:131-44.

2. Shimada H, Sakakima H, Tsuchimochi K, et al. Senescence of chondrocytes in aging articular cartilage: GADD $45 \beta$ mediates p21 expression in association with $\mathrm{C} / \mathrm{EBP} \beta$ in senescence-accelerated mice. Pathol Res Pract 2011;207:225-31.

3. Unterluggauer H, Hampel B, Zwerschke W, JansenDürr P. Senescence-associated cell death of human endothelial cells: the role of oxidative stress. Exp Gerontol 2003;38:1149-60.

4. Khan IM, Gilbert SJ, Caterson B, Sandell LJ, Archer CW. Oxidative stress induces expression of osteoarthritis markers procollagen IIA and 3B3(-) in adult bovine articular cartilage. Osteoarthritis Cartilage 2008;16:698-707.

5. Chainiaux F, Magalhaes JP, Eliaers F, Remacle J, Toussaint O. UVB-induced premature senescence of human diploid skin fibroblasts. Int J Biochem Cell Biol 2002;34:1331-9.

6. Gorgoulis VG, Pratsinis H, Zacharatos P, et al. p53dependent ICAM-1 overexpression in senescent 
human cells identified in atherosclerotic lesions. Lab Invest 2005;85:502-11.

7. Severino J, Allen RG, Balin S, Balin A, Cristofalo VJ. Is beta-galactosidase staining a marker of senescence in vitro and in vivo? Exp Cell Res 2000;257:162-71.

8. Dimri GP, Lee X, Basile G, et al. A biomarker that identifies senescent human cells in culture and in aging skin in vivo. Proc Natl Acad Sci USA 1995;92:9363-7.

9. Kurz DJ, Decary S, Hong Y, Erusalimsky JD. Senescence-associated (beta)-galactosidase reflects an increase in lysosomal mass during replicative ageing of human endothelial cells. J Cell Sci 2000;113:3613-22.

10. Alberti KG, Zimmet P, Shaw J. Metabolic syndrome-a new world-wide definition. A Consensus Statement from the International Diabetes Federation. Diabet Med 2006;23:469-80.

11. McClelland AD, Kantharidis P. microRNA in the development of diabetic complications. Clin Sci (Lond) 2014;126:95-110.

12. Won HY, Park JB, Park EY, Riew KD. Effect of hyperglycemia on apoptosis of notochordal cells and intervertebral disc degeneration in diabetic rats. J Neurosurg Spine 2009;11:741-8.

13. Park EY, Park JB. Dose- and time-dependent effect of high glucose concentration on viability of notochordal cells and expression of matrix degrading and fibrotic enzymes. Int Orthop 2013;37:1179-86.

14. Park EY, Park JB. High glucose-induced oxidative stress promotes autophagy through mitochondrial damage in rat notochordal cells. Int Orthop 2013; 37:2507-14

15. Mobbs RJ, Newcombe RL, Chandran KN. Lumbar discectomy and the diabetic patient: incidence and outcome. J Clin Neurosci 2001;8:10-3.

16. Sakellaridis N. The influence of diabetes mellitus on lumbar intervertebral disk herniation. Surg Neurol 2006;66:152-4.

17. Simpson JM, Silveri CP, Balderston RA, Simeone FA, An HS. The results of operations on the lumbar spine in patients who have diabetes mellitus. J Bone Joint Surg Am 1993;75:1823-9.

18. Zhao CQ, Wang LM, Jiang LS, Dai LY. The cell biology of intervertebral disc aging and degeneration. Ageing Res Rev 2007;6:247-61.

19. Gruber HE, Watts JA, Hoelscher GL, et al. Mitochondrial gene expression in the human annulus: in vivo data from annulus cells and selectively harvested senescent annulus cells. Spine J 2011;11:782-91.

20. Roberts S, Evans EH, Kletsas D, Jaffray DC, Eisenstein SM. Senescence in human intervertebral discs. Eur Spine J 2006;15 Suppl 3:S312-6.

21. Gruber HE, Ingram JA, Norton HJ, Hanley EN Jr. Senescence in cells of the aging and degenerating intervertebral disc: immunolocalization of senescenceassociated beta-galactosidase in human and sand rat discs. Spine (Phila Pa 1976) 2007;32:321-7.

22. Kletsas D. Senescent cells in the intervertebral disc: numbers and mechanisms. Spine J 2009;9:677-8.

23. Kim KW, Chung HN, Ha KY, Lee JS, Kim YY. Senescence mechanisms of nucleus pulposus chondrocytes in human intervertebral discs. Spine J 2009;9:658-66.

24. Kim KW, Lim TH, Kim JG, Jeong ST, Masuda K, An HS. The origin of chondrocytes in the nucleus pulposus and histologic findings associated with the transition of a notochordal nucleus pulposus to a fibrocartilaginous nucleus pulposus in intact rabbit intervertebral discs. Spine (Phila Pa 1976) 2003;28:98290.

25. Baynes JW. Role of oxidative stress in development of complications in diabetes. Diabetes 1991;40:405-12.

26. Nishikawa T, Araki E. Impact of mitochondrial ROS production in the pathogenesis of diabetes mellitus and its complications. Antioxid Redox Signal 2007;9:343-53.

27. Dorn GW 2nd. Mechanisms of non-apoptotic programmed cell death in diabetes and heart failure. Cell Cycle 2010;9:3442-8.

28. Victor VM, Rocha M, Herance R, Hernandez-Mijares A. Oxidative stress and mitochondrial dysfunction in type 2 diabetes. Curr Pharm Des 2011;17:3947-58.

29. Brandl A, Hartmann A, Bechmann V, Graf B, Nerlich $\mathrm{M}$, Angele P. Oxidative stress induces senescence in chondrocytes. J Orthop Res 2011;29:1114-20.

30. Furukawa A, Tada-Oikawa S, Kawanishi S, Oikawa S. $\mathrm{H} 2 \mathrm{O} 2$ accelerates cellular senescence by accumulation of acetylated p53 via decrease in the function of SIRT1 by NAD+ depletion. Cell Physiol Biochem 2007;20:45-54.

31. Ben-Porath I, Weinberg RA. The signals and pathways activating cellular senescence. Int J Biochem Cell Biol 2005;37:961-76.

32. Campisi J. Cellular senescence as a tumor-suppressor mechanism. Trends Cell Biol 2001;11:S27-31. 